\title{
Comportamiento sexual del macho cabrío murciano granadino en distintas edades
}

\author{
Galián Arnaldos, S.; Peinado Ramón, B.; Poto Remacha, Á. y Almela Veracruz, L.
}

Instituto Murciano de Investigación y Desarrollo Agrario y Alimentario. Departamento de Acuicultura y Tecnología de la Producción Animal. Murcia. España.

\section{PaLABRAS ClAVE}

Calidad seminal.

Caprino.

Libido.

Testaje.

\section{RESUMEN}

Se ha evaluado el comportamiento sexual de machos cabríos de raza Murciano Granadina de diferentes edades para comprobar los tiempos de reacción y la capacidad de monta, y compararlos con la calidad seminal de cada macho, el momento de aparición de la libido y el primer eyaculado válido. Se utilizaron 18 machos con edades entre los 3 meses y los 7 años. Semanalmente se midió el tiempo que tardaban en montar y el número de servicios en una mañana. Se midió volumen y concentración espermática total y al primer eyaculado de la mañana se le evaluó su calidad seminal, valorando calidad de movimiento individual y porcentaje de espermatozoides móviles. Los resultados muestran que machos jóvenes presentaron menor tiempo de reacción y mayor capacidad de monta en una mañana que machos mayores de 1 año, con menor volumen de eyaculado y concentración espermática. Los machos mostraron un aumento del tiempo de reacción conforme aumenta el número de montas, mayor a partir del tercer servicio. El volumen de eyaculado y la concentración espermática tienden a disminuir en cada salto. La calidad seminal de los machos jóvenes fue aumentando a lo largo de las semanas, y la motilidad individual y porcentaje de espermatozoides móviles no se vieron afectados por los sucesivos eyaculados en una misma mañana. En definitiva, la edad presenta gran variabilidad en los parámetros medidos.

\section{Sexual behaviour of murciano granadina bucks at different ages}

\section{SUMMARY}

The sexual behavior of male goats of the Murciano Granadina breed at different ages has been evaluated to check reaction times and mounting capacity, and compare them with the seminal quality of each male, the moment of appearance of libido and the first valid ejaculate. Eighteen males with ages between 3 months and 7 years were used. The time it took to set up and the number of services in one morning were measured weekly. Total sperm volume and concentration were measured and the first ejaculate in the morning was evaluated for seminal quality, assessing individual movement quality and percentage of motile sperm. The results show that young males have shorter reaction time and greater mounting capacity in one morning than males older than 1 year, with lower ejaculate volume and sperm concentration. Males show an increase in reaction time as the number of mounts increases, greater after the third service. The ejaculate volume and sperm concentration act to decrease with each jump. The seminal quality of the young males was increasing throughout the weeks, and the individual motility and percentage of motile spermatozoa were not affected by the successive ejaculates in the same morning. In short, age shows great variability in the parameters measured.

INFORMATION

Cronología del artículo.

Recibido/Received: 30.07 .2020 .

Aceptado/Accepted: 20.11 .2020

On-line: 15.04.2021.

Correspondencia a los autores/Contact e-mail:

laura.almela@carm.es

\section{INTRODUCTION}

El éxito económico de una explotación vendrá determinado en gran medida por el nacimiento del mayor número de ejemplares posible, y entre otros factores, este éxito reproductivo dentro de un rebaño dependerá de que los machos utilizados como sementales proporcionen elevadas tasas de preñez durante la época de cubrición. Para lograr esto, lo ideal es que un macho combine una buena capacidad de producir espermatozoides viables con el deseo de detectar y cubrir a las hembras en celo (Mateos, 1990). Un macho con baja actividad sexual va a presentar problemas tanto en recogida con vagina artificial (más tiempo de reacción) como en el porcentaje de hembras cubiertas por monta natural, y si no se identifica a tiempo puede retrasar o comprometer el rendimiento productivo del rebaño (Pérez y Mateos, 1993). Sin olvidar que el nú- 
mero de hembras que un macho pueda dejar preñadas durante la estación de cubrición dependerá también de su producción espermática y de la calidad de dicha producción, al igual que el número de dosis que podremos obtener de un eyaculado cuando se le quiera incluir en un programa de inseminación artificial (Pérez, 1992). Ningún trabajo ha demostrado claramente que exista una buena correlación entre alguna de las características de un eyaculado y la capacidad de servicio del macho que lo produjo. Vijil et al. (1985) no encontraron correlación entre calidad seminal y parámetros de comportamiento sexual (tiempo de reacción y eficacia de cubrición) en moruecos manchegos y karakul adultos. Tampoco la encontraron en toros Mateos (1978) y Chenoweth (1986), quienes publicaron que deben evaluarse calidad seminal y comportamiento sexual por separado, pero todos los autores coinciden en afirmar que el conjunto de las mismas determina su fertilidad (Pérez, 1992) por lo que no solo las características seminales son importantes, también lo es el número de hembras cubiertas y el número total de cubriciones realizadas, siendo lo ideal que una buena calidad seminal se acompañe de una libido elevada. Un factor que no debemos olvidar es la precocidad de la llegada a la pubertad de los machos, ya que, si esta capacidad de cubrición y calidad seminal adecuada aparece pronto en el ejemplar, este podrá ser testado e incorporado a los programas de mejora genética mucho antes, alargando su vida productiva.

Siempre que se evalúe el comportamiento sexual de los machos se deben tener en cuenta los diferentes factores que van a determinar dicho comportamiento: variaciones individuales entre machos, que pueden llegar a ser muy marcadas, edad del macho, raza, estación del año ya que estas especies son reproductores estacionales y existen diferencias de comportamiento por la influencia del fotoperiodo (Folch y Roca, 1982; Mateos y Zubieta, 1990). En la raza que nos ocupa, la Murciano Granadina, la estacionalidad es muy poco marcada, o incluso inexistente (Zarazaga et al. 2003; ovigen.es), por lo que no tuvimos en cuenta los meses del año en los que se desarrolló este estudio.

El comportamiento sexual de machos cabríos ha sido estudiado por algunos autores (Chemineau 1986, Zubieta 1990, Pérez et al. 1992, Pérez, 1992) y en general todos utilizan como parámetros objetivos la libido, el tiempo de reacción a la primera eyaculación y el número de cubriciones en un tiempo dado. Relacionado con este tiempo de reacción (tiempo que transcurre desde que el macho toma contacto con la hembra hasta que realiza la primera cubrición), están los actos de cortejo, con los que el macho investiga el estado fisiológico de la hembra. Estos actos suelen incluir (Espinosa et al. 2014):

Golpes con la cabeza y manoteos, el macho toca a la hembra con golpes de su cabeza y sus extremidades delanteras repetidamente.

\section{Olfateos ano-genitales de la otra cabra.}

Micción, en el caso de los machos adultos se orinan en su propia cara y barbas con el pene extendido.
Flehmen generalmente tras olfatear la orina del otro animal el macho muestra la boca abierta, el labio superior levantado con exposición de las encías, con la cabeza y el cuello extendido.

Cortejo, el macho da pasos hacia delante haciéndole cabriolas a la hembra

Monta: el macho coloca sus patas delanteras en la parte superior del dorso de la hembra, puede haber o no movimientos pélvicos.

Los machos de raza Murciano Granadina también realizan el lengüeteo cuando se acercan por los flancos y a la cabeza de la hembra.

El objetivo de este trabajo fue estudiar el comportamiento sexual (tiempo de reacción y capacidad de cubrición) de machos cabríos de raza Murciano Granadina de diferentes edades, la producción y calidad seminal de su eyaculado y la precocidad de la libido.

\section{MATERIALY MÉTODOS}

Evaluamos el comportamiento sexual de 18 machos cabríos de raza Murciano Granadina de diferentes edades. Se obtuvieron un total de 260 observaciones, de las cuales, 167 fueron eyaculados y 93 fueron intentos en los que los distintos machos no saltaron sobre la hembra. Diez machos tenían menos de 1 año durante el transcurso del estudio y 8 machos eran mayores de 1 año. Todos los machos se alojaban en corrales individuales de $10 \mathrm{~m}^{2}$, con una superficie cubierta y un patio exterior de ejercicio. Su alimentación consistió en un $800 \mathrm{~g}$ por animal y día de un concentrado de piensos comerciales a base de cereales y proteínas vegetales y $2 \mathrm{Kg}$ por animal y día de paja de cereales y alfalfa (Medicago sativa). Estas cantidades se depositaban en los comederos una vez al día y el animal disponía de ellos a su gusto. En total la dieta de cada macho cabrío se compuso de aproximadamente $2800 \mathrm{Kcal} /$ día y unos $250 \mathrm{~g}$ de proteína digestible por día. Cada macho dispuso siempre de agua ad libitum. El estudio se realizó una vez por semana durante 14 semanas, entre los meses de julio y octubre de 2019. Los machos eran entrenados una vez por semana. Estudiamos el comportamiento sexual midiendo el tiempo de reacción de cada macho (tiempo que empleaba en saltar sobre la hembra) y la capacidad de cubrición (el número de montas que realizaba en una misma mañana), observando si realizaba los pasos previos de cortejo a la hembra. En los machos más jóvenes se estudió también a qué edad mostraban interés por la hembra, saltaban sobre ella y eyaculaban, evaluándose al microscopio óptico a 100 aumentos (Leica DFC420) la calidad de estos primeros eyaculados.

El semen de cada eyaculado se recogió mediante vagina artificial para caprino. A cada eyaculado obtenido se le midió el volumen y la concentración espermática mediante un espectrofotómetro (SpermaCue, Minitüb) con el fin de estudiar si estos parámetros diferían sustancialmente con el número de saltos. Al eyaculado del primer salto se le evaluó también su calidad, manteniéndolo en un baño termostático a $37^{\circ} \mathrm{C}$ y estudiando la motilidad individual, en la cual se valoró subjetivamente el movimiento espermático en 
una escala de 0 a 5 , donde 0 indica no movimiento y 5 movimiento progresivo muy rápido (Cabodevila y Catena, 2012) y el porcentaje de espermatozoides móviles (porcentaje subjetivo de espermatozoides con movimiento visualizados en un campo al microscopio óptico a 100 aumentos, considerándose el total del campo como el 100\%).

Para la realización del estudio se utilizó una hembra inmovilizada. El tiempo de reacción se midió con un cronómetro desde que el macho entraba al corral con la hembra hasta que eyaculaba. Se concedió un tiempo de 5 minutos máximo para la monta, si tras estos 5 minutos no se había producido el salto a la hembra, se sacaba al macho del corral, anotando el correspondiente fallo. Este proceso se repitió con cada macho, dejando unos 20 minutos entre salto y salto, tantas veces como saltos diera el animal. Tras dos intentos sin salto, no se volvía a probar con ese macho.

Durante el tiempo que cada macho estaba con la hembra se observó si dedicaba tiempo a la conducta sexual típica del cortejo de los caprinos.

\section{RESULTADOS}

Al observar a los machos más jóvenes, vimos grandes diferencias en su comportamiento sexual. Comenzaron a ser estudiados cuando contaban con 3 meses de edad, inicio del comportamiento sexual primario (monta sin eyaculación). A los 4,5 meses obtuvimos eyaculado de un macho, pero con pocos espermatozoides y todos inmóviles. Este mismo macho consiguió una valoración de 4 en la motilidad individual y $85 \%$ de móviles cuando se le recogió semen dos meses después, al contar con 6,5 meses de edad. Otros tres machos de 4,5 meses también saltaban sobre la hembra, pero no producían aún eyaculado. Un macho produjo espermatozoides con una calidad de movimiento de 2 y un porcentaje de espermatozoides móviles de $20 \%$ a los 3,5 meses. Otros tres machos de edades entre 3 y 6 meses no llegaron a saltar ninguna semana del estudio o lo hacían de forma esporádica. En general, los machos menores de 1 año mostraron comportamientos sexuales muy diversos, desde aquellos que saltaban con regularidad sobre la hembra y eyaculaban sin dificultad a partir de los 4,5 meses, otros que comenzaban más tarde, entre los 6 y 8 meses y aquellos que mostraban libido, pero no montaban a la hembra (exteriorizando el pene e incluso eyaculando). Hubo machos que saltaban a la hembra, pero sin llegar a eyacular y otro grupo que no mostraba libido ni interés por la monta.

Obtuvimos los promedios del tiempo en segundos que se tardaba en montar a la hembra en cada uno de los saltos, los del volumen de eyaculado y la concentración espermática total para cada uno de los saltos. Estos parámetros se calcularon para todos los machos a la vez, sin distinguir por edad (Tabla I), y comparando entre machos menores y mayores de un año (Tabla II).

Los resultados fueron sometidos a análisis estadístico mediante el programa Statgraphics ${ }^{\circledR}$ centurión. Se analizaron los promedios de cada parámetro mediante la prueba-F en la tabla de ANOVA para determinar
Tabla I. Promedio del tiempo de salto (seg), volumen de eyaculado $(\mathrm{mL})$ y concentración espermatozoides en el eyaculado $\left(\times 10^{6}\right)$ en cada uno de los saltos, sin diferenciar por edad de los machos (Average jump time (sec), ejaculate volume $(\mathrm{mL})$ and sperm concentration in the ejaculate $\left(x 10^{6}\right)$ in each of the jumps, without differentiating by age from the males).

\begin{tabular}{lcll}
\hline & Tiempo de salto & $\begin{array}{l}\text { Volumen } \\
\text { eyaculado } \\
0,77^{\mathrm{a}} \pm 0,07\end{array}$ & $\begin{array}{l}\text { Concentración } \\
\text { total spz } \\
3640^{\mathrm{a}} \pm 310\end{array}$ \\
$\begin{array}{l}\text { Salto 1 } \\
(\mathrm{N}=52)\end{array}$ & $54,9^{\mathrm{a}} \pm 6,7$ & $0,69^{\mathrm{a}} \pm 0,08$ & $3041^{\mathrm{a}, \mathrm{b}} \pm 302$ \\
$\begin{array}{l}\text { Salto 2 } \\
(\mathrm{N}=41)\end{array}$ & $58,9^{\mathrm{a}} \pm 7,4$ & $0,61^{\mathrm{a}} \pm 0,07$ & $2325^{\mathrm{b}, \mathrm{c}} \pm 295$ \\
$\begin{array}{l}\text { Salto 3 } \\
(\mathrm{N}=30)\end{array}$ & $74,5^{\mathrm{a}} \pm 10,5$ & & \\
$\begin{array}{l}\text { Salto } 4 \\
(\mathrm{~N}=22)\end{array}$ & $121,6^{\mathrm{b}} \pm 16$ & $0,63^{\mathrm{a}} \pm 0,09$ & $1949^{\mathrm{c}} \pm 234$ \\
$\begin{array}{l}\text { Salto 5 } \\
(\mathrm{N}=17)\end{array}$ & $152,7^{\mathrm{b}, \mathrm{c}} \pm 22,2$ & $0,5^{\mathrm{a}} \pm 0,09$ & $1515^{\mathrm{c}} \pm 204$ \\
$\begin{array}{l}\text { Salto 6 } \\
(\mathrm{N}=5)\end{array}$ & $169,3^{\mathrm{c}} \pm 33,1$ & $0,52^{\mathrm{a}} \pm 0,12$ & $1861^{\mathrm{b}, \mathrm{c}} \pm 359$ \\
\hline
\end{tabular}

Promedio \pm error estándar. Letras distintas en la misma columna indican diferencias significativas. $\mathrm{N}=$ número de eyaculados de cada salto.

si hay diferencias significativas entre las medias. Las pruebas de rangos múltiples determinaron qué medias fueron significativamente diferentes de otras, con un nivel del $95 \%$ de confianza.

En general se aprecia una tendencia al aumento del tiempo que tarda un macho en saltar conforme aumenta el número de saltos. Comparando por edades, el tiempo de reacción de machos jóvenes fue significativamente inferior que el de los adultos, en los saltos 1,2 y 3. Los test estadísticos no nos dan diferencias significativas en el tiempo de reacción del resto de saltos, pero estos siguen siendo inferiores en machos menores de un año que en mayores. Otra diferencia que aparecía por edad, es que los machos menores de 1 año fueron capaces de saltar un mayor número de veces en la misma mañana (un macho joven llegó a saltar 7 veces en una mañana y 6 jóvenes saltaron 6 veces en varias de las semanas de la prueba, aunque no siempre proporcionaban eyaculados) mientras que los mayores de un año saltaron como máximo 5 veces (machos de 1.5, 4 y 5 años de edad). Los machos de 6 y 7 años dieron un máximo de 2 saltos en la misma mañana, habiendo mañanas en las que no saltaron ni una sola vez. Apreciamos por tanto mayor libido en machos jóvenes o adultos jóvenes, con respecto a machos cabríos que ya tienen 6 y 7 años de edad.

En este trabajo medimos el volumen y la concentración espermática obtenidos en el eyaculado de cada salto. Uniendo los resultados de todos los machos, sin distinguir entre grupos de edad, obtuvimos los valores promedio para los parámetros volumen de eyaculado de la Tabla I.

El volumen de eyaculado disminuye en promedio en cada salto, siendo el primer eyaculado de la mañana el de mayor cantidad, no existiendo diferencias signifi- 
cativas entre ellos. El aumento del volumen medio del salto 6 puede explicarse debido el pequeño número de muestras que teníamos para calcular el promedio de ese salto.

Si analizamos el volumen de eyaculado diferenciando a los machos por grupos de edad (menores y mayores de un año, Tabla II), obtuvimos que el volumen de eyaculado fue inferior en los machos más jóvenes con respecto a los mayores, siendo estas diferencias significativas en los saltos 2, 3, 4 y 5 . En el salto 1 el volumen es menor en los machos menores de un año, pero sin diferencia significativa estadística. Lo mismo ocurre con el salto 6, aunque en este caso puede deberse al escaso número de valores que tenemos para analizar este salto.

También hay una tendencia general a la disminución de la concentración de espermatozoides totales en el eyaculado (Tabla I), siendo significativamente mayor la concentración del primer salto que en los saltos 3, 4, 5 y 6. El aumento de la concentración espermática en el salto 6 con respecto al salto 5 puede deberse a que los escasos valores con los que hacer el promedio, ya que un bajo número de machos llegaron a saltar 6 veces. Si diferenciamos por edad (Tabla II) vemos que los machos menores de 1 año presentan concentraciones espermáticas inferiores a los mayores, siendo significativas estas diferencias en los saltos 1,2, 3 y 4 . La concentración del salto 6 no se consideró para análisis estadístico por contar con solo una medida en el caso de los machos mayores de 1 año.

Se evaluó de forma subjetiva la calidad seminal del primer eyaculado de la mañana, midiéndose la motilidad individual de cada muestra (escala de 0 a 5) y el porcentaje de espermatozoides móviles. Para determinar a qué edad esta calidad seminal era óptima para realizar ensayos de inseminación artificial, se dividió a los machos en distintos grupos de edad, desde los 3.5 meses hasta los 7 años. Los resultados se muestran en la Tabla III. En general, la calidad seminal es óptima para inseminación artificial desde los 5 meses, cuando obtenemos un 4 en motilidad individual y más de un $50 \%$ de espermatozoides móviles. La menor calidad de las muestras de 9 y 10 meses se debe a variabilidad entre machos expuestos a temperaturas estivales, ya que dos machos de estas edades presentaban calidades inferiores a las del promedio.

La calidad seminal de los machos menores de un año se evaluó también en los diferentes saltos, para determinar si ésta variaba en los sucesivos eyaculados. Los resultados se muestran en la Tabla IV.

Estos resultados nos muestran que al contrario de lo que ocurre con el volumen de eyaculado y la concentración espermática (ambas disminuyen con los sucesivos saltos), la calidad seminal se mantiene, no habiendo diferencias significativas entre las calidades espermáticas de los de los eyaculados obtenidos en los distintos saltos a lo largo de la mañana.

Teniendo en cuenta todos estos resultados, vemos que la libido y la capacidad de monta en un mismo día es mayor en machos menores de 1 año que en los mayores, pero que el volumen de eyaculado y la concentración espermática son mayores en los adultos. Pudimos apreciar que los machos adultos dedicaban mayor tiempo a realizar las pautas de cortejo que los machos más jóvenes, pero con el paso del tiempo a lo largo de las semanas en los que se realizó este trabajo, vimos que los machos jóvenes alargaban el tiempo de cortejo a la hembra, incorporando pateos y olfateos, aunque siendo todavía este cortejo más breve que el de machos adultos. Al estudiar la calidad seminal obtuvimos diferencias debidas a la edad del ejemplar, así como variaciones entre individuos. Como tendencia general, apreciamos que la calidad seminal es menor en los eyaculados de los machos jóvenes que en la de los adultos, pero esta calidad fue aumentando conforme pasaban las semanas del estudio, pareciéndose cada vez más a la de machos adultos.

Tabla II. Promedio del tiempo de salto (seg.), volumen de eyaculado (mL) y concentración espermatozoides en el eyaculado $\left(\times 10^{6}\right)$ en cada uno de los saltos, diferenciando entre machos mayores de 1 año y menores (Average jump time (sec.), ejaculate volume $(\mathrm{mL})$ and sperm concentration in the ejaculate $\left(\times 10^{6}\right)$ in each of the jumps, differentiating between males over 1 year and younger).

\begin{tabular}{|c|c|c|c|c|c|c|}
\hline & \multicolumn{2}{|c|}{ Tiempo de Salto } & \multicolumn{2}{|c|}{ Volumen eyaculado } & \multicolumn{2}{|c|}{ Concentración spz } \\
\hline & $<1$ año & >1 año & $<1$ año & >1 año & $<1$ año & >1 año \\
\hline $\begin{array}{l}\text { Salto } 1 \\
N=23 ; N=29\end{array}$ & $42,6^{a} \pm 6,5$ & $81,6^{b} \pm 14,1$ & $0,69^{a} \pm 0,08$ & $0,91^{a} \pm 0,13$ & $3075^{a} \pm 335$ & $4620^{b} \pm 559$ \\
\hline $\begin{array}{l}\text { Salto } 2 \\
N=21 ; N=20\end{array}$ & $44,8^{\mathrm{a}} \pm 7,7$ & $87,06^{b} \pm 13,8$ & $0,46^{a} \pm 0,05$ & $1,15^{\mathrm{b}} \pm 0,17$ & $2476^{a} \pm 266$ & $4580^{b} \pm 688$ \\
\hline $\begin{array}{l}\text { Salto } 3 \\
N=18 ; N=12\end{array}$ & $62,8^{a} \pm 11,1$ & $102,18^{b} \pm 22,3$ & $0,42^{\mathrm{a}} \pm 0,04$ & $1,14^{b} \pm 0,16$ & $1888^{a} \pm 180$ & $4730^{b} \pm 1100$ \\
\hline $\begin{array}{l}\text { Salto } 4 \\
N=12 ; N=10\end{array}$ & $113,9^{a} \pm 20,8$ & $140,22^{a} \pm 21$ & $0,39^{\mathrm{a}} \pm 0,04$ & $1,14^{\mathrm{b}} \pm 0,18$ & $1667^{\mathrm{a}} \pm 227$ & $2966^{\mathrm{b}} \pm 519$ \\
\hline $\begin{array}{l}\text { Salto } 5 \\
N=10 ; N=7\end{array}$ & $141,2^{a} \pm 24,6$ & $190^{\mathrm{a}} \pm 74$ & $0,34^{\mathrm{a}} \pm 0,06$ & $0,92^{\mathrm{b}} \pm 0,12$ & $1381^{\mathrm{a}} \pm 201$ & $2320^{b} \pm 580$ \\
\hline $\begin{array}{l}\text { Salto } 6 \\
N=4 ; N=1\end{array}$ & $193,2 \pm 34,5$ & $61,5^{*}$ & $0,42 \pm 0,07$ & $0,95^{*}$ & $1606 \pm 286$ & $3900^{*}$ \\
\hline
\end{tabular}




\section{DISCUSIÓN}

La calidad seminal, la capacidad de servicio y la precocidad en la llegada a la pubertad son factores que van a determinar el valor de un macho cabrío como reproductor. En relación con la llegada a la pubertad, se ha publicado que depende de la raza y la condición corporal del animal, Ángel et al., 2020 han publicado que machos de la raza Alpino presentan la pubertad a los 6,5 meses, mientras que en los machos Criollos del subtrópico mexicano fue a los 4,3 meses (evaluada esta como la aparición de la primera célula espermática viable) y machos Nubio de Alabama (Estados Uni-

Tabla III. Promedio de motilidad individual y $\%$ de espermatozoides móviles del primer eyaculado de la mañana, en los distintos rangos de edad (Average individual motility and \% of motile sperm of the first ejaculate in the morning, in the different age ranges).

\begin{tabular}{lcc}
3,5 meses & 2 & Motilidad Individual \\
\% meses & $4,12^{\mathrm{a}} \pm 0,24$ & $20^{\star}$ \\
6 meses & $4,04^{\mathrm{a}} \pm 0,2$ & $58,7^{\mathrm{a}, \mathrm{b}} \pm 8,8$ \\
7 meses & $3,31^{\mathrm{b}} \pm 0,24$ & $63,3^{\mathrm{b}} \pm 7,2$ \\
8 meses & $4,31^{\mathrm{a}} \pm 0,24$ & $52,5^{\mathrm{a}, \mathrm{b}} \pm 8,8$ \\
9 meses & $3,31^{\mathrm{b}} \pm 0,24$ & $70,0^{\mathrm{b}, \mathrm{c}, \mathrm{d}} \pm 8,8$ \\
10 meses & $3,37^{\mathrm{a}, \mathrm{b}} \pm 0,24$ & $51,9^{\mathrm{a}, \mathrm{b}} \pm 8,8$ \\
1,5 años & $4,03^{\mathrm{a}} \pm 0,18$ & $37,5^{\mathrm{a}} \pm 8,8$ \\
4 años & $4,1^{\mathrm{a}} \pm 0,14$ & $69,3^{\mathrm{b}, \mathrm{c}} \pm 6,7$ \\
5 años & $4,25^{\mathrm{a}} \pm 0,28$ & $78,3^{\mathrm{d}} \pm 5,1$ \\
6 años & $3,98^{\mathrm{a}} \pm 0,13$ & $77,0^{\mathrm{c}, \mathrm{d}} \pm 7,9$ \\
7 años & $4,06^{\mathrm{a}} \pm 0,28$ & $74,7^{\mathrm{c}, \mathrm{d}} \pm 4,9$ \\
\hline
\end{tabular}

Promedio \pm error estándar. Letras distintas en la misma columna indican diferencias significativas; *Solo una medida, no se considera para análisis estadístico.

Tabla IV. Promedio de la calidad seminal de los sucesivos eyaculados en machos menores de 1 año (Average seminal quality of successive ejaculates in males under 1 year of age).

\begin{tabular}{lll}
\hline & Mot. individual & $\%$ spz. móviles \\
Eyaculado 1 $(\mathrm{N}=23)$ & $3,93^{\mathrm{a}} \pm 0,16$ & $61,47^{\mathrm{b}} \pm 4,13$ \\
Eyaculado 2 $(\mathrm{N}=21)$ & $3,96^{\mathrm{a}} \pm 0,16$ & $66,53^{\mathrm{b}} \pm 4,01$ \\
Eyaculado $3(\mathrm{~N}=18)$ & $4,27^{\mathrm{a}} \pm 0,2$ & $72,5^{\mathrm{b}} \pm 4,92$ \\
Eyaculado 4 $(\mathrm{N}=12)$ & $4,11^{\mathrm{a}} \pm 0,21$ & $71,13^{\mathrm{b}} \pm 5,14$ \\
Eyaculado 5 $(\mathrm{N}=10)$ & $4^{\mathrm{a}} \pm 0,21$ & $66,36^{\mathrm{b}} \pm 5,14$ \\
Eyaculado 6 $(\mathrm{N}=4)$ & $3,5^{\mathrm{a}} \pm 0,48$ & $70^{\mathrm{b}} \pm 12$
\end{tabular}

Promedio \pm error estándar. Letras diferentes en la misma columna indican diferencias significativas. dos), la iniciaban a los 4,5 meses (Skalet et al., 1988). Los machos de raza Murciano Granadina evaluados mostraron capacidad de monta desde los 4,5 meses de edad, pero sin espermatozoides viables hasta los 5,5-6 meses de vida, aunque se observó gran variabilidad entre ejemplares (uno de ellos ya dio espermatozoides viables a los 3,5 meses) tanto en comportamiento como en calidad seminal. Uno de los machos jóvenes igualaba a los 6 meses en motilidad individual y porcentaje de espermatozoides móviles a los mayores de un año, aunque presentó menor volumen de eyaculado y concentración espermática, y entre los 7-8 meses de edad, 4 machos jóvenes presentaron una calidad seminal óptima para inseminación artificial y regularidad en la monta a la hembra. Hubo tres machos de edades entre 6 y 8 meses que todavía no mostraron una monta regular.

En todos los rangos de edad estudiados se observa una fuerte variabilidad individual entre los distintos machos, por lo que es conveniente conocer las características individuales de cada ejemplar antes de seleccionarlo como reproductor, de acuerdo con lo publicado por Pérez, 1992. El número de saltos realizados por los machos de más de cinco años es insuficiente para su mantenimiento en programas de inseminación y testaje, por lo que la edad óptima para la producción de semen estaría por debajo de los cinco años y por encima de los 4,5 meses.

Los resultados nos muestran que tanto el volumen de eyaculado como la concentración espermática aumentan con la edad de los machos, lo que coincide con lo encontrado con otros autores en la misma raza caprina Murciano Granadina (Zubieta (1990); Roca et al. (1991a)) En otras razas como la Jamunapari (Shina et al., 1981), La Mancha (Muhuyi et al. 1982) Y Nubiana (Skalet et al. 1988) una mayor calidad seminal también apareció al aumentar la edad de los machos. Según Evans y Maxwell (1987), para realizar una inseminación artificial un eyaculado debe tener una concentración espermática no inferior a 2,500×106 espermatozoides $/ \mathrm{ml}$, por lo que con nuestros resultados podría utilizarse el primer eyaculado de los machos menores de un año, similar a lo encontrado por Roca et al. (1991b) quienes publicaron que machos de 9-10 meses de edad eran aptos para ser usados en programas reproductivos, aunque en el presente estudio la edad de los machos para entrar en programas de testaje se disminuye a los 6,5 meses con un máximo de ocho. En machos de edad superior podían utilizarse hasta el $4^{\circ}$ eyaculado de la mañana. Los parámetros de motilidad individual y \% de móviles no fueron significativamente diferentes entre machos jóvenes y de más edad, lo que también los hace aptos para su uso en programas de reproducción. Los valores obtenidos para la motilidad individual y la concentración total de espermatozoides para machos menores y mayores de 1 año es acorde con la encontrada por Roca et al. (1991b).

Las circunstancias ambientales también parecen afectar a la producción de semen, y más concretamente durante el tiempo de estío, observándose una disminución de la calidad en cuanto al número de espermatozoides vivos y su calidad de movimiento (Tabla III, machos con 9 y 10 meses). Aunque coincidimos con 
Zarazaga et al. 2003 y otros muchos autores, en que el periodo de luz-oscuridad en la raza Murciano Granadina no influye en la producción seminal, las altas temperaturas del estío (en los meses de julio y agosto de 2019 se registraron temperaturas medias de 28,5 y $27,9^{\circ} \mathrm{C}$ respectivamente, llegándose a alcanzar temperaturas máximas de más de $38^{\circ} \mathrm{C}$, incluso sobrepasando los $40^{\circ} \mathrm{C}$ ) sí han producido disminución de calidad (Pérez, 1992), si las comparamos con las de septiembre y octubre que tuvieron una media de 23,8 y $19,8^{\circ} \mathrm{C}$ y cuyos máximos quedaron siempre por debajo de los $34^{\circ} \mathrm{C}$ (Siam, Imida). Especialmente afectados por las elevadas temperaturas estivales se vieron dos machos jóvenes, lo que hizo disminuir tanto el promedio de motilidad individual como el porcentaje de espermatozoides móviles.

En cuanto a las diferencias en los tiempos de reacción, inferiores en machos jóvenes, son debidas a la falta de actos de cortejo, o a la brevedad de estos. Algunos autores achacan a la inexperiencia de los machos jóvenes y la competencia con machos adultos la falta de las pautas en el comportamiento sexual previo a la monta (Ungerfeld y González, 2009; Orihuela, 2014). Machos dominantes, de mayor corpulencia y fortaleza que los jóvenes pueden atacar a estos últimos si le dedican un tiempo considerable al cortejo previo a la monta, perdiendo los machos jóvenes la posibilidad de montar a las hembras del conjunto del rebaño, por lo que la estrategia de estos machos sería la rapidez en la cópula, limitando al mínimo o incluso ignorando el tiempo de cortejo.

\section{CONCLUSIONES}

De este trabajo podemos concluir que en cuanto al comportamiento sexual de machos cabríos de raza Murciano Granadina, los ejemplares jóvenes presentan menor tiempo de reacción y mayor capacidad de servicio que los adultos.

El tiempo de reacción tan corto en los machos jóvenes se debe al instinto de montar hembra, ya que si realizan el cortejo serán acometidos por los adultos con mayores defensas y capacidad de acometida.

La calidad seminal de machos menores de un año es suficiente para ser utilizados como reproductores, aunque existe gran variabilidad entre ejemplares. Esta calidad va aumentando conforme aumenta la edad del macho, a la vez que van adquiriendo la conducta sexual propia de la especie e incorporándola al cortejo de la hembra.

Los mejores machos cabríos para utilizar como sementales serían aquellos en los que la edad ya permita una calidad seminal óptima y presenten un deseo sexual para detectar y cubrir las hembras en celo de su rebaño.

\section{AGRADECIMIENTOS}

Este trabajo ha sido financiado por Fondos Europeos de Desarrollo Regional. FEDER 1420 - 26. Con el proyecto titulado: Los recursos genéticos animales resilientes al cambio climático. Mejora y diversificación de productos alimenticios derivados y obtenidos de forma biosostenible.

\section{BIBLIOGRAFÍA}

Ángel, O, Veliz, FG, Carrillo, E, y Rivas, R 2020, Determinación del inicio de la pubertad en machos cabríos de la raza alpino nacidos en Invierno en el subtrópico mexicano.(on line) https://bmeditores. $\mathrm{mx} /$ ganaderia/determinacion-del-inicio-de-la-pubertad-en-machoscabrios-de-la-raza-alpino-nacidos-en-invierno-en-el-subtropicomexicano/\#: : text=Los\%20resultados $\% 20$ del\%20presente $\% 20$ estudio,caprinos\%20Criollos\%20de\%20esta\%20regi\%C3\%B3n.

Cabodevila, J, y Catena, M2012, Evaluación de semen bovino congelado www.engormix.com/ganaderia-carne/articulos/evaluacion-semenbovino-congeleado-t29765. htm.

Chemineau, P 1986, Sexual behavior and gonadal activity during the year in the tropical Creole meat goat. Male mating behavior, testis diameter, ejaculate characteristics and fertility. Reprod. Nutr. Dévelop. 26 (2A), 453-460.

Chenoweth, PH 1986, Libido testing. En: Current therapy in theriogenology. D.A. Morrow. ED. WB. Saunders Company. Philadelphia. USA. 136-142.

Espinosa, R, Córdoba, A, y Soto, R 2014, Comportamiento sexual en ovinos y caprinos. https://www.researchgate.net/publication/265784418

Evans, G, y Maxwell, WMC 1987, Salamon's artificial insemination of sheep and goats. Butterworth and Co. Sydney. 194pp.

Folch, J, y Roca, M 1982, Características sexuales del morueco de RasaAragonesa durante el primer año de vida. Influencia de la época de nacimiento. Ann. INIA. Vol.16, pp. 99.

Mateos, E 1978. Breeding soundness, sexual behaviour and fertility in Range Bulls. Tesis Master of Science. Department of Physiology and Biophysics. Colorado State University. Fort Collins, Colorado.

Mateos, E 1990, Avances en reproducción caprina. Mundo Ganadero, 9, págs. 41-51.

Mateos, E, y Zubieta, M 1990, Influencia del estímulo sexual en los niveles de testosterona plasmática de machos cabríos. [Influence of sexual stimulation on plasmatic testosterone levels in male goats]. Investigación agraria. Producción y sanidad animales-INIA(España).5(3), 109-1 19.

Muhuyi, W, Drobnis, EZ, Nelson, T, y Lin, Y 1982, Season, breed and age influences on production and freezability of dairy goat semen. Int. Conf. Goat. Tucson.

Orihuela, A 2014, La conducta sexual del carnero: Revisión. Revista mexicana de ciencias pecuarias, 5(1), 49-89. http://www.scielo.org. $\mathrm{mx} /$ scielo.php? script=sci_arttext\&pid=S2007-1 1242014000100004

Pérez, B 1992, Estudio de los parámetros de valoración del rendimiento reproductivo en macho cabrío de las razas Verata y Malagueña (Doctoral dissertation, Thesis, Veterinary School, Complutense University, Madrid, Spain, 200pp). https://eprints.ucm. es/3208/1/T18230.pdf

Pérez, B, Mateos Rex, E, y Pintado, B 1992, Seasonal influence on sexual behavior in bucks. Abstracts. V International Conference on Goats: March, 1992. 274.

Pérez, B, y Mateos, E 1993, Evaluación del comportamiento sexual en machos cabríos. Mundo Ganadero, 1, $1 \mathrm{~T}$.

Roca, J, Martínez, E, Vázquez, JM, Ruíz, S, y Coy, P 1991a, Influence of season on testicle size and libido in male goats from the Mediterranean area. Anim. Prod. 52, 317-321.

Roca, J, Martínez, E, Vázquez, JM, Ruíz, S, y Coy, P 1991b, Influencia de la edad sobre los parámetros reproductivos de machos cabríos de raza Murciano-Granadina. Arch.Zootec.40:173-179.

Shina, NK, Wani, GM, y Sahni, KL 1981, Effect of seasons and age on seminal atributes of Jamunapari buck. Indian Vet. J., 58 (12): 963-965.

Skalet, LH, Rodrigues, HD, Goyal, HO, Maloney, MM, y Noble, RC 1988, Effects of age and season on the type and occurrence of sperm abnormalities in Nubian bucks. Am.J. Vet.Res., 49 (8): 1284-1289. 
Ungerfeld R, y González, SP 2009, Social dominance and courtship and mating behavior in rams in non-competitive and competitive pen tests. Reprod Domes Anim;44(1):44-47.

Vijil, E, Gonzalo, C, Ciudad, C, y Ruiz, J 1985, Jerarquía social, diámetro testicular, libido y calidad seminal en los morvecos de raza Manchega y Karakul. ITEA. 60, págs. 19-27.
Zarazaga, L, Guzmán, J, y Pérez, M 2003, Estacionalidad reproductiva en caprino y su relación con las concentraciones plasmáticas de melatonina. Seoc. 218-221.

Zubieta, M 1990, Evolución y valoración de la actividad funcional en machos reproductores caprinos durante su desarrollo. M. Thesis. IAMZ. Zaragoza.

https://ovigen.es/razas/raza-murciano-granadina/

http://siam.imida.es/apex/f?p=101:1:2776738701152138 